() 2015 IEEE. Personal use of this material is permitted. Permission from IEEE must be obtained for all other uses, in any current or future media, including reprinting/republishing this material for advertising or promotional purposes, creating new collective works, for resale or redistribution to servers or lists, or reuse of any copyrighted component of this work in other works.

\title{
Are Converged Access Networks suitable in rural areas?
}

\author{
Ana Muñoz Díaz ${ }^{\S *}$, Carmen Mas Machuca*, Senior Member IEEE \\ ${ }^{\S}$ Universidad Politécnica de Madrid, Spain \\ *Technische Universität München, Arcisstraße 21, Munich, Germany \\ \{ana.munoz,cmas\}@tum.de
}

\begin{abstract}
Rural areas are the last regions aiming at getting access to broadband services due to the high investments and limited revenues. However, new architectures such as Hybrid Passive Optical Network (HPON) allow interconnecting fixed users and Base Stations with the same infrastructure. This paper looks into the advantages of the HPON joint planning with respect to an independent planning of mobile backhaul and fixed users. The recently proposed HPON architecture could offer more bandwidth to the base stations (10Gbps) and less bandwidth to residential users (300-500Mbps). The results show the cost reduction of supporting joint planning in two real rural areas and analyze the impact of the number of base stations and their inter BS distance (i.e. different cell sizes) on the savings.
\end{abstract}

Keywords: convergence, NGOA, PON, broadband access, rural areas

\section{INTRODUCTION}

Communications are being increasingly developed all around the world during the last years. Gigabit PON (GPON) is currently the widest deployed optical access network for dense urban fixed users, delivering up to $80 \mathrm{Mbps}$ (for 1:32 power splitter). Access network operators are facing the challenge of providing their fixed users with higher bandwidths (300-500Mbps) in 2020.

On the other hand, most of the mobile backhauls are based on Point-to-Point (P2P) optical architectures interconnecting the base stations to the central offices. In this way, each base station has full capacity but installing its optical fibre requires important investment. Furthermore, the fast mobile technology evolution (from $2 \mathrm{G}$, to $3 \mathrm{G}$ and $4 \mathrm{G}$ ) is offering higher bandwidth per user implying a fast upgrade of the backhaul. This upgrade affects the number and location of the base stations, but also the possibility to install microcells at some locations to improve the coverage. These microcells should also be connected to the central office (i.e. new fibre infrastructure is required).

The evolution of optical technologies allows the required increase of bandwidth but also offers longer reach from central office to optical network units. The increase of maximum reach allows operators to reduce the number of central offices (so-called node consolidation) and hence, promising savings to operators.

Next Generation Optical Access (NGOA) networks are solutions offering higher bandwidth, longer reach and larger client counts [1]. One of the most promising NGOA networks is the TWDM PON, which combines TDM and WDM multiplexing techniques. This solution offers different bandwidth to the end users depending on where they are connected to, within the access network. Hence, it is possible to use the same optical access network infrastructure to connect fixed users requiring $300 \mathrm{Mbps}$ as well as base stations requiring $10 \mathrm{Gbps}$. The planning of such a network is referred in this paper to as joint planning and it will be compared with a disjoint planning of legacy access networks as well as disjoint planning of NGOA networks.

Last but not least, NGOA networks have been studied in dense urban areas [2,3] where there is a higher demand of services, higher concentration of users and therefore, higher expected revenues. However, rural areas have a sparse and low user distribution which requires high investments and promises limited revenues. This fact, however, is being compensated nowadays by new programs (e.g. Horizon 2020 Digital Agenda [4]) aiming at offering a fair broadband access distribution even in rural areas.

The goal of this paper is to evaluate the potential savings of joint planning of NGOA networks in rural networks. This paper is structured as follows: Section 2 presents the optical access network architectures considered for this study. Section 3 introduces the main steps performed in order to implement the planning tool that allowed the evaluation and comparison of the different planning scenarios presented in Section 4 together with the results obtained. Finally, conclusions are gathered in Section 5.

\section{OPTICAL ACCESS NETWORK ARCHITECTURES}

In this section, the considered architectures along the study, both existing and NGOA, are described.

\subsection{Gigabit-PON (GPON)}

Based on the ITU-T Recommendations G.984.x, GPON is the widest deployed Fibre To The Home/Building (FTTH/B) access architecture connecting business and residential users in Europe. GPON is based on a point-tomultipoint topology, in which a passive power splitter is placed at the remote node, between the Central Office (CO) and the subscribers. Data rates of up to 2.5 Gbps for the downstream and upstream directions can be 
provided. Common splitting ratios for this architecture are 1:32 or 1:64, being the most widely deployed 1:32, with a maximum passive reach of $20 \mathrm{~km}$. Regarding wavelength ranges, GPON works within ranges from 1480 $\mathrm{nm}$ to $1500 \mathrm{~nm}$ for the downstream direction and from $1260 \mathrm{~nm}$ to $1360 \mathrm{~nm}$ for the upstream. Moreover, as wavelengths are fixed, tunable receivers and transmitters are not required. This architecture is depicted in Figure $1(\mathrm{a})$.

\subsection{Point-to-point (P2P)}

This architecture shown in Figure 1(b) refers to a communication scheme in which two end nodes (CO and Base Station (BS) in this case) are connected directly, with no multiplexing steps performed between them. Nowadays, P2P connections are used in most of the mobile backhaul networks. This architecture can offer high capacity to the base stations and, in addition, the same wavelengths can be used, encouraging costs reduction in transponders through the application of economy of scale.

\subsection{TWDM PON}

In this model proposed by the OASE Project [1], two stages of remote nodes are considered to connect businness and residential users in order to benefit from the advantages of two different multiplexing techniques. In the first stage, Wavelength Division Multiplexing (WDM) access is made possible through the usage of Arrayed Wavelength Gratings (AWGs) at the first remote node. AWG creates independent wavelength channels for each end user. Common number of wavelengths $(\Lambda)$ is $40 \lambda$ and $80 \lambda$. At the second remote node, passive power splitters are used to perform Time Division Multiplexing (TDM) of each wavelength, which allows sharing the wavelength capacity among different users. In general, splitting ratios $N$ of 1:16 and 1:32 are considered when planning FTTH/B networks. A graphical representation of this architecture is shown in Figure 1(c). With this new proposal, longer distances to the end users are allowed as well as a higher client count $(\Lambda . N)$. In addition, higher data rates are provided to the end users with the chance of offering different capacities to each of them.

\subsection{Hybrid PON (HPON)}

This last architecture is based on the TWDM PON but exploiting the advantage of joint planning, that is, BS are directly connected to the AWGs and consequently, getting the full wavelength capacity. Hence, in this architecture, fixed users are connected to a power splitter and getting $10 \mathrm{Gbps} / \mathrm{N}$ capacity, whereas BS are getting 10Gbps. The major advantage of this new proposal depicted in Figure 1(d) is that infrastructure and equipment can be shared between fixed users and BS, reducing the total investment required.

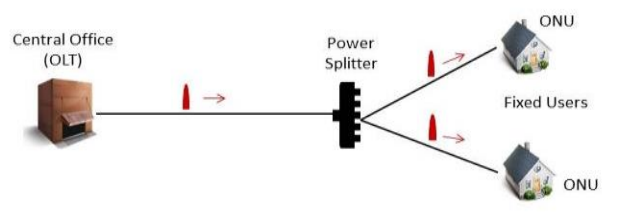

a) GPON Architecture connecting fixed users

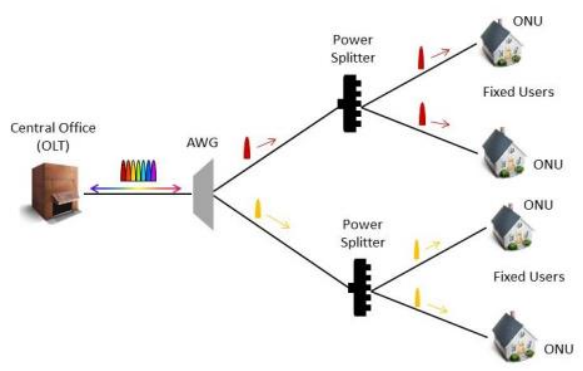

c) TWDM PON connecting fixed users

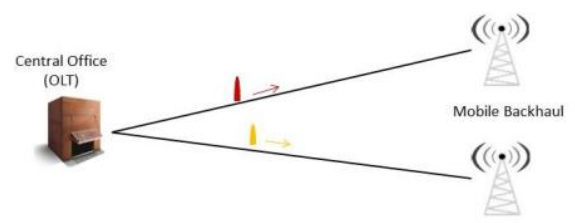

b) P2P Architecture connecting $B S$

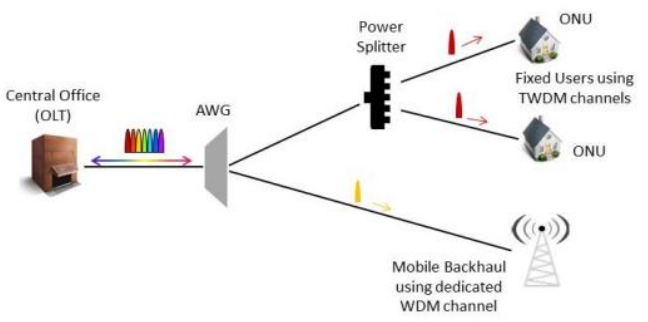

d) HPON connecting fixed users and BS

Figure 1. Optical Access Network Architectures

\section{METHODOLOGY: MODELLING APPROACH}

Let us introduce in this section the methodology to evaluate each of the scenarios in rural areas and some hints of its implementation using OpenStreetMap and Matlab.

\subsection{Gathering Area Information}

The geographic information required for this study is obtained from OpenStreetMap (OSM), software that allows free access and distribution of geographic data. The maps can be downloaded and edited with Java OSM Editor. The selected area contains information of interest for the network planning, such as buildings, streets, 
nodes; and it has to be parsed to an appropriate Matlab format. Further preparation of the data base is carried out, such as location of intersection nodes, or association of buildings to the nearest street.

\subsection{Distribution of Base Stations (BS)}

In order to determine the location of the BS, hexagonal sectors are considered to design an "ideal grid" in which BSs are homogeneously distributed over the map. Depending on the given inter BS distance, a certain area has a larger or lower number of BS. However, this ideal location of BS is not always realistic since it can fail on a river, in a forest, etc. Hence, the location of the BS is updated and associated to the nearest street node (as confirmed by an operator that BSs are located on the ground and next to a road in rural areas).

\subsection{Routing}

Before routing can be performed, clustering is needed in order to group fixed users and assign them to power splitters, or group power splitters (and BS in HPON) and assign them to AWGs. Clustering is performed by using the "k-means" method. This method also gives the centroid location of each cluster, which is associated to the nearest intersection node (it was assumed that power splitters and AWGs are placed at intersection nodes). Once the clusters are defined, the fibre layout by routing is computed. The objective is the minimization of total investment, which is driven by duct trenching and installation costs [2,3]. For this purpose, a modification of Dijkstra's algorithm is proposed, in which the weight associated to a street section that has already been used for routing is reduced, encouraging this way duct sharing along the paths. Finally, due to the larger distances in rural areas which may exceed the maximum reach, the limitations and cost increases caused by the purchase and placement of Reach Extenders (RE) are taken into account. An algorithm that analyses the distance from the CO to each building and BS has been implemented in order to place the required RE in an optimal position.

\section{SCENARIOS AND CASE STUDY}

\subsection{Scenarios}

The different scenarios which result from combining the different optical access network architectures with different splitting rations $(\Lambda$ and $N)$ are the following:

- "GPON Disjoint" scenario considers GPON 1:32 to connect the fixed users to the CO and P2P to connect the BS to the CO. Hence the delivered capacity is $78 \mathrm{Mbps} /$ fixed users and $10 \mathrm{Gbps} / \mathrm{BS}$.

- "TWDM Disjoint" considers TWDM PON for fixed users and P2P for the BS. Four different TWDM PONs are studied (using $(\Lambda, N)):(40,16),(80,16),(40,32),(80,32)$. The delivered capacity is $625 \mathrm{Mbps} /$ fixed users (when $N=16$ ), $312 \mathrm{Mbps} /$ fixed users (when $N=32$ ), and 10Gbps/BS.

- "HPON Joint" considers HPON for both fixed users and BS. The same 4 different $(\Lambda, N)$ combinations are considered and the delivered bandwidth is the same as for "TWDM PON Disjoint".

\subsection{Case Study 1: Impact of Architecture}

This study is performed in the rural area of Miesbach, Germany, which covers $\sim 17 \mathrm{~km}^{2}$ and 2682 buildings, which are sparsely distributed around the area. In this first case study, the impact of splitting ratios for the different TWDM PON architectures is examined. Equipment and infrastructure required for the different combinations of splitting ratios are depicted in Figure 2. In Figure 2(a) is shown how the number of PS depends only on their own splitting ratios, due to the fact that the number of buildings is constant in the area; meanwhile the number of AWGs depends not only on their own wavelengths but also on the number of PS connected to them. On the other hand, it can be observed that, for this scenario, RE were only required in the last architecture in which maximum reach allowed is much lower $(\sim 7 \mathrm{~km})$. On the other hand and regarding Figure 2(b), it can be appreciated how duct is clearly reduced in comparison with fibre for every considered architecture.

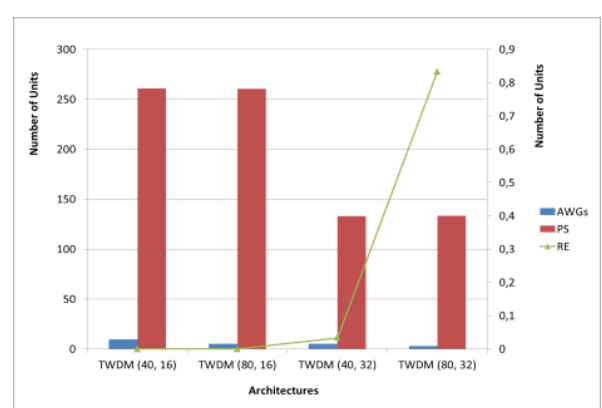

a) Splitters and Reach Extenders

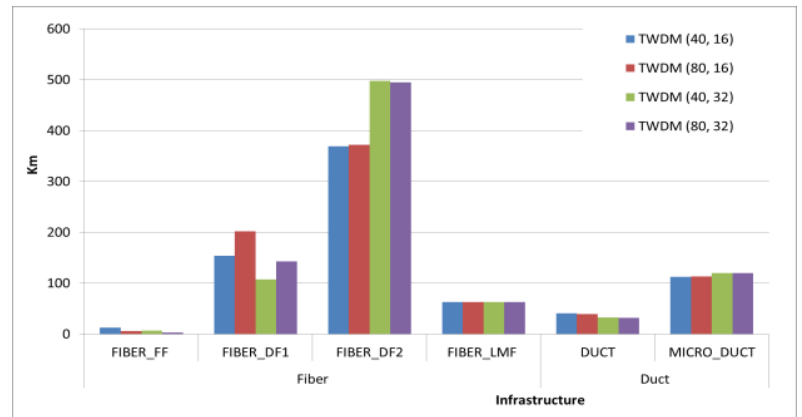

b) Fibre and Duct Layout

Figure 2. Equipment and Infrastructure required for the different TWDM PON Architectures 


\subsection{Case Study 2: TWDM Disjoint vs. HPON Joint}

Let us compare the total costs between TWDM Disjoint and HPON Joint planning (depicted in Figure 3). It can be observed the total savings for both alternatives and for two different inter BS distances $(0.5$ and $1 \mathrm{~km})$. Furthermore, the study is carried out in two different scenarios: the first one refers to the scenario described previously in section $4.2\left(17 \mathrm{~km}^{2}\right.$ and $\sim 160$ buildings $\left./ \mathrm{km}^{2}\right)$; meanwhile the second region represents a larger area around Miesbach $\left(\sim 65 \mathrm{~km}^{2}\right)$ with a lower building density of $\sim 40 \mathrm{buildings} / \mathrm{km}^{2}$. The savings are significantly higher for larger areas with lower building density where more BS benefit from the joint planning by sharing the infrastructure required by the fixed users. This is also reflected by the higher savings for shorter inter BS distances (hence, larger number of BS in the area).

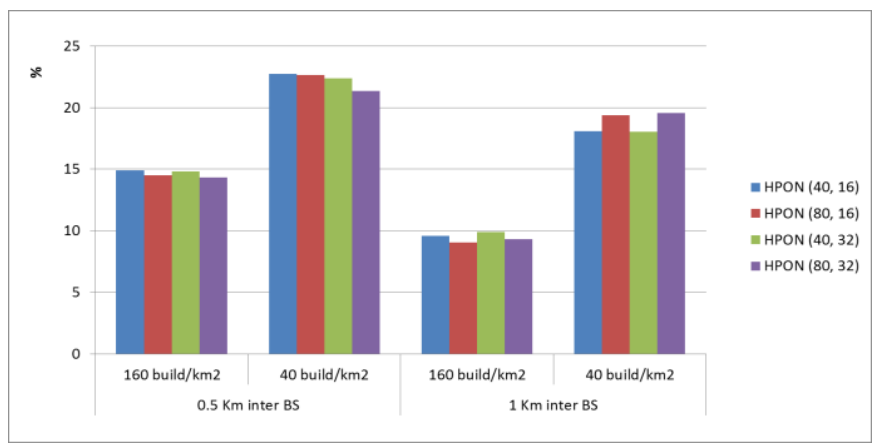

Figure 3. Savings (\%) from HPON respect to TWDM PON for different building densities and inter BS distances

\subsection{Case Study 3: From GPON towards HPON}

The total costs of the different planning scenarios are depicted in Figure 4(a). The costs are given in Cost Units (CU) which is the cost of a GPON ONU. It can be observed that costs do not vary significantly based on the splitting rations. In all cases, TWDM has an $8 \%$ higher total investment with respect to GPON. However, when looking at the cost per delivered bandwidth shown in Figure 4(b), it has been proved that TWDM requires significant lower cost/Gbps per end user than GPON since it offers much higher BW at similar cost. Furthermore HPON offers significant savings not only in total cost but also cost/Gbps, becoming this way a promising solution for operators in rural areas.

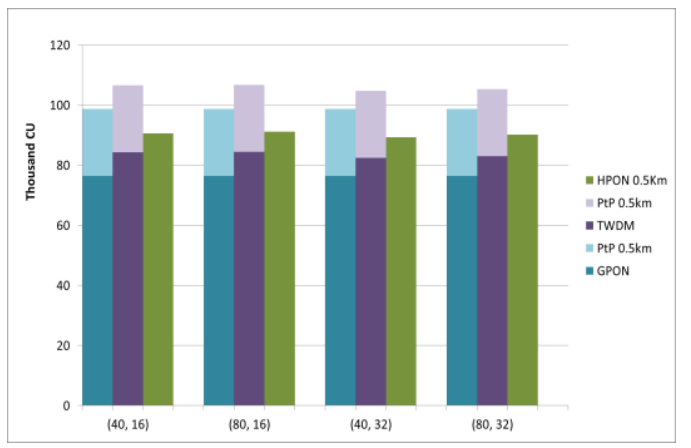

a) Total costs comparison

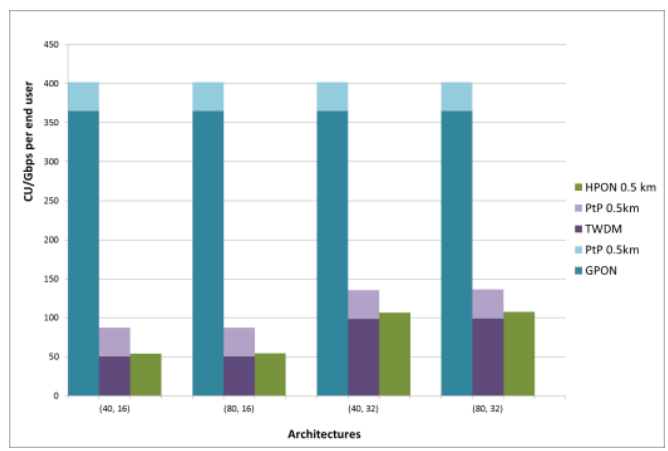

b) [CU/Gbps] per end user

Figure 4. Comparison between the scenarios proposed in section 4.1

\section{CONCLUSIONS}

This paper shows the advantage of using joint planning when using TWDM PON solutions. Joint planning allows operators to roll out optical fibre and use it to interconnect base stations and fixed users. The best solution HPON $(40,16)$ requires lower investment than GPON while delivering 300Mbps/fixed user.

\section{REFERENCES}

[1] OASE Project: http://www.ict-oase.eu/

[2] M. Rahman et al. "Advantages of Joint Access Network Planning in Dense Populated Areas", in Proc. NOC 2014, Milan, Italy, June 2014.

[3] C. Mas Machuca et al. "Cost savings dependence on base station inter-distance in converged access network planning of dense populated areas", in Proc. ICTON 2014, Austria, 2014.

[4] Digital Agenda Europe: http://ec.europa.eu/digital-agenda/en/information-communication-technologieshorizon-2020 
